\title{
Inhibition of return to occluded objects
}

\author{
DO-JOON YI \\ Yale University, New Haven, Connecticut \\ MIN-SHIK KIM \\ Yonsei University, Seoul, Korea \\ and \\ MARVIN M. CHUN \\ Yale University, New Haven, Connecticut
}

\begin{abstract}
Since many visual objects are vulnerable to occlusion, an active process that tracks objects behind occluders confers considerable ecological validity to the visual system. We studied this possibility by testing whether inhibition of return can be observed with occluded objects. In our experiments, two moving objects disappeared or reappeared behind occluders while a cue and a probe were presented. Contrary to the results of a previous study (Tipper, Weaver, Jerreat, \& Burak, 1994), responses were consistently delayed for the cued object that was occluded when it was cued (Experiment 1), when it was probed (Experiment 2), or both (Experiment 3). These results suggest that attention can select occluded objects that are out of view. Our findings are in line with prior studies that have demonstrated similar perceptual/attentional effects for occluded objects, as well as for visible objects.
\end{abstract}

The response of detecting the onset of a target is slower for a previously searched location than for novel locations, a delay called inhibition of return (IOR). According to Posner and Cohen (1984), IOR allows search mechanisms to favor new locations that have not yet been examined, thereby maximizing search efficiency (see also Klein, 1988). The IOR effect is strongest when attention is summoned to a peripheral location in an exogenous manner (Posner \& Cohen, 1984) and when it is accompanied by oculomotor programming (Rafal, Calabresi, Brennan, \& Sciolto, 1989). These findings suggest that IOR is closely linked to the system that coordinates attention and eye movements (see Abrams \& Dobkin, 1994, and Klein, 2000, for reviews).

For typical static displays consisting of two or more peripheral locations around a central fixation point, the locus of inhibition in the visual field does not change following an eye movement, indicating that IOR operates with an environment-based coordinate system, rather than with retinotopic coordinates (Maylor, 1985; Posner \& Cohen, 1984). However, visual scenes in the environment

A portion of this work was presented at the 23rd European Conference on Visual Perception, Groningen, Netherlands, 2000. This work was supported by a Brain Neuroinformatics Research Program Grant from the Korean Ministry of Science and Technology to M.-S.K. and, in part, by National Science Foundation Grant BCS-0096178 to M.M.C. We thank Kimron Shapiro, Raymond Klein, Steven Tipper, and two anonymous reviewers for their helpful comments and suggestions on the draft, as well as Keetaek Kham for programming advice. Correspondence concerning this article should be addressed to D.-J. Yi, Department of Psychology, Yale University, 2 Hillhouse Avenue, P.O. Box 208205, New Haven, CT 06520-8205 (e-mail: dojoon.yi@yale.edu). not only are static, as in the case of an office with a stationary desk, but also are dynamic, as in that of a scene of moving people in a crowded airport. When one looks for a friend in an airport in which there are many people moving around, IOR based on static environmental coordinates would not be useful. For example, if IOR were only environment based, attention might be inefficiently redirected to a previously attended person who had moved into a new location, while missing a target friend that had moved into a previously attended location. Ecologically speaking, the IOR mechanism needs to incorporate more sophisticated object-oriented components (Tipper, Driver, \& Weaver, 1991; Tipper, Weaver, Jerreat, \& Burak, 1994).

Accordingly, Tipper and his colleagues (Tipper et al., 1991; Tipper et al., 1994) have demonstrated that IOR operates with both environment-based and object-based coordinates. In their experiments, two peripheral boxes moved around a central fixation box. During the orbiting motion, a cue appeared at one of the peripheral boxes, followed by a second cue at the central box. These two cues captured visual attention, causing it to engage toward and then disengage away from the cued peripheral box. After the two outer boxes had moved to new locations, a probe appeared at the cued or uncued locations or at the cued or uncued boxes that had moved to new locations. The subjects' reaction times (RTs) to the probe were longer for the cued than for the uncued location, which demonstrates environment-based IOR (Maylor, 1985; Posner \& Cohen, 1984). The new finding was that the RTs were longer for probes in the cued box than for those in the uncued box, indicating that the inhibitory mechanism tracked the cued box to the new location in 
an object-based manner. This result suggests that IOR facilitates search by adopting an environment-based mode, an object-based mode, or both (Abrams \& Dobkin, 1994; Gibson \& Egeth, 1994; Tipper et al., 1991; Tipper et al., 1994).

The dynamic object-based property of IOR raises an intriguing question: Can we observe IOR for an object that is invisible behind an occluder? This question can be rephrased in two specific ways. First, if attention is allocated to an object while it is out of sight behind an occluder, is it still possible to observe IOR when the object reappears? Second, does an inhibitory bias against a previously attended object remain attached to it even after the object disappears behind an occluder? Occlusion prevails everywhere around us. At the airport, a person may or may not be seen when blocked by other objects, such as people, columns, advertisement signs, and so forth. Since visual objects are occluded so frequently, an objectoriented visual mechanism should be robust across brief occlusion to enjoy ecological validity. Indeed, previous studies on occlusion perception have provided evidence that occluded objects have perceptual/attentional effects on the visual system that are similar to those for visible objects. For example, an object that disappeared behind an illusory Kanizsa figure persisted long enough to influence perceived apparent motion (Yantis, 1995). A separate study showed that when one end of a rectangle was cued, attention spread across an invisible part of the rectangle behind an occluder in a way that facilitated probe discrimination at the other end of the rectangle (Moore, Yantis, \& Vaughan, 1998). Finally, attentive tracking for multiple objects was not impaired when moving objects were intermittently occluded (Scholl \& Pylyshyn, 1999). These findings indicate that the visual system maintains representations for occluded objects in the environment. In fact, amodal completion of an occluded object appears to be somewhat obligatory, so that the visual system cannot help but represent objects behind occluders (Davis \& Driver, 1994, 1998; He \& Nakayama, 1992).

However, initial evidence suggests that IOR cannot tag occluded visual representations. Tipper et al. (1994, Experiment 6) examined whether cuing an occluded object would reveal effects of attention when the object reappeared into view. Two static boxes and two moving boxes were presented around a fixation box. A size difference was introduced between the static and the moving boxes as a depth and occlusion cue, and relative size was manipulated between groups. The moving boxes were larger than the static boxes for the moving-near group, and vice versa for the static-near group. For both groups, large boxes looked closer to observers than did small ones, so that a large box was perceived to occlude a small box when they overlapped. In the task, a moving box moved toward a static box, and as the moving box occluded the static one, a peripheral cue appeared to induce visual attention. After a brief delay, a central cue appeared at fixation to draw attention away from the peripherally cued location/object. The moving boxes con- tinued to migrate to new locations, whereupon a probe appeared either in a cued or an uncued box or in a cued or an uncued location. Whereas environment-based IOR was observed for both groups, object-based IOR was observed only for the moving-near group. That is, if moving boxes disappeared behind static boxes in the staticnear group, IOR did not occur at the new location of the cued moving box. On the basis of these results, Tipper et al. (1994) concluded that it is necessary for an object to be visible when cued to produce object-based IOR.

Tipper et al.'s (1994) finding is not compatible with the previously mentioned studies on occlusion perception. If the representation of an invisible object is maintained behind an occluder (Yantis, 1995) and is given attention (Moore et al., 1998; Scholl \& Pylyshyn, 1999), the visual system should be able to attach inhibitory tags to occluded objects, revealing object-based IOR. We suspect that Tipper et al. may have missed evidence for IOR to the occluded object because visual attention was allocated to the occluding object rather than to the occluded object. In such a circumstance, the inhibitory tag would be attached only to the previously attended, occluding object. Their findings may have resulted from two aspects of their methods. First, the static occluding boxes were as salient as the moving ones. Both the static and the moving boxes were well-defined rectangles with distinctive colors. Second, the static boxes were as task relevant as the moving ones because, on half of the trials, the static boxes per se were directly cued when they were not occluding a moving box at the same time. Thus, it is possible that on the trials in which the static box was cued while occluding a moving box, the occluding static box captured attention and, accordingly, no IOR occurred to the occluded moving box.

In the present study, therefore, we reconsidered the possibility that an inhibitory tag can be attached to an occluded object. To test this hypothesis, we revised Tipper et al.'s (1994) experiment so as to maximize attentional allocation to the occluded objects. First, we reduced the visual salience of the occluders. Second, the occluders were never task relevant. Cues appeared on the occluders only when the moving object was concurrently occluded underneath them. Third, accretion and deletion animation features were used to enhance the perception of moving objects' disappearing and reappearing behind occluders (Scholl \& Pylyshyn, 1999). Finally, we used 64 frames for $360^{\circ}$ of object movement around the center, which resulted in smoother motion animations than Tipper et al.'s (1994) 32-frame rendition. All of these manipulations served to enhance the perceptual representations of occluded objects and, thus, increase the probability of IOR for them.

\section{EXPERIMENT 1}

We tested whether IOR can occur to an object that undergoes occlusion when cued. If the object out of sight is perceived as occluded, so that a cue in the same location directs visual attention to the persisting representa- 
tion, IOR should occur to a probe on the object when it reappears. In contrast, if the object must be visible for object-based IOR, probe RTs should not be different for cued and uncued occluded objects, as Tipper et al. (1994) reported.

A sample trial sequence is shown in Figure 1. Two squares, positioned across from each other on opposite sides of the screen around the fixation square, moved clockwise or counterclockwise along a circular path. Two diagonal quadrants were covered with two separate occluders, so that the moving squares disappeared and reappeared behind them with appropriate deletion and accretion animation. The first cue always appeared at the location where one of the moving squares was occluded. Then the second cue appeared at the fixation square, to disengage attention from the cued location while the objects were still moving. Finally, a probe showed up after the moving squares had reappeared and moved to new locations. By comparing RTs at probed locations or objects, we were able to determine whether location- and object-based IOR had occurred.

\section{Method}

Subjects. Eighteen undergraduates at Yonsei University, Seoul, Korea, participated for course credit. All had normal or corrected- to-normal vision. Each subject was tested in an individual booth dimly lit with indirect incandescent lighting. None of the subjects knew the purpose of the experiment or the expected results beforehand. The entire experiment took less than $30 \mathrm{~min}$.

Apparatus. The experiment was conducted using MATLAB equipped with psychophysics toolbox extensions (Brainard, 1997) on an IBM-compatible computer and a monitor with a $75-\mathrm{Hz}$ refresh rate. Viewing distance was fixed to $40 \mathrm{~cm}$, using a chinrest. A standard keyboard was used as the response device.

Stimuli. All the stimuli were presented against a black background. Three hollow squares, outlined in dark gray and diagonally subtending a visual angle of $1.9^{\circ}$, were used as the two moving objects and the fixation mark. Moving squares lay opposite each other across the fixation square, with an eccentricity of $6.5^{\circ}$. Each occluder was fan shaped with a central angle of $90^{\circ}$ and was marked with a pair of lines, each of which was light gray and $3.1^{\circ}$ long. These occluders were placed on two diagonal quadrants: the upper right and the lower left quadrants or the upper left and lower right quadrants. The two cues and the probe were all disks with a diameter of $1.7^{\circ}$. The cues were colored white, and the probe was blue. Both the cues and the probes were presented along the motion path in the center of a quadrant. The shortest distance between them and the boundary of an occluder was $4.3^{\circ}$.

To animate the movement of the two peripheral squares, a series of frames was presented successively. The position of each square changed by $5.625^{\circ}$ in polar angle between each successive frame, so that 64 frames were required for the $360^{\circ}$ orbiting movement. Each frame was replaced by the next frame every $40 \mathrm{msec}$, without an interframe interval. When moving objects disappeared or reap-

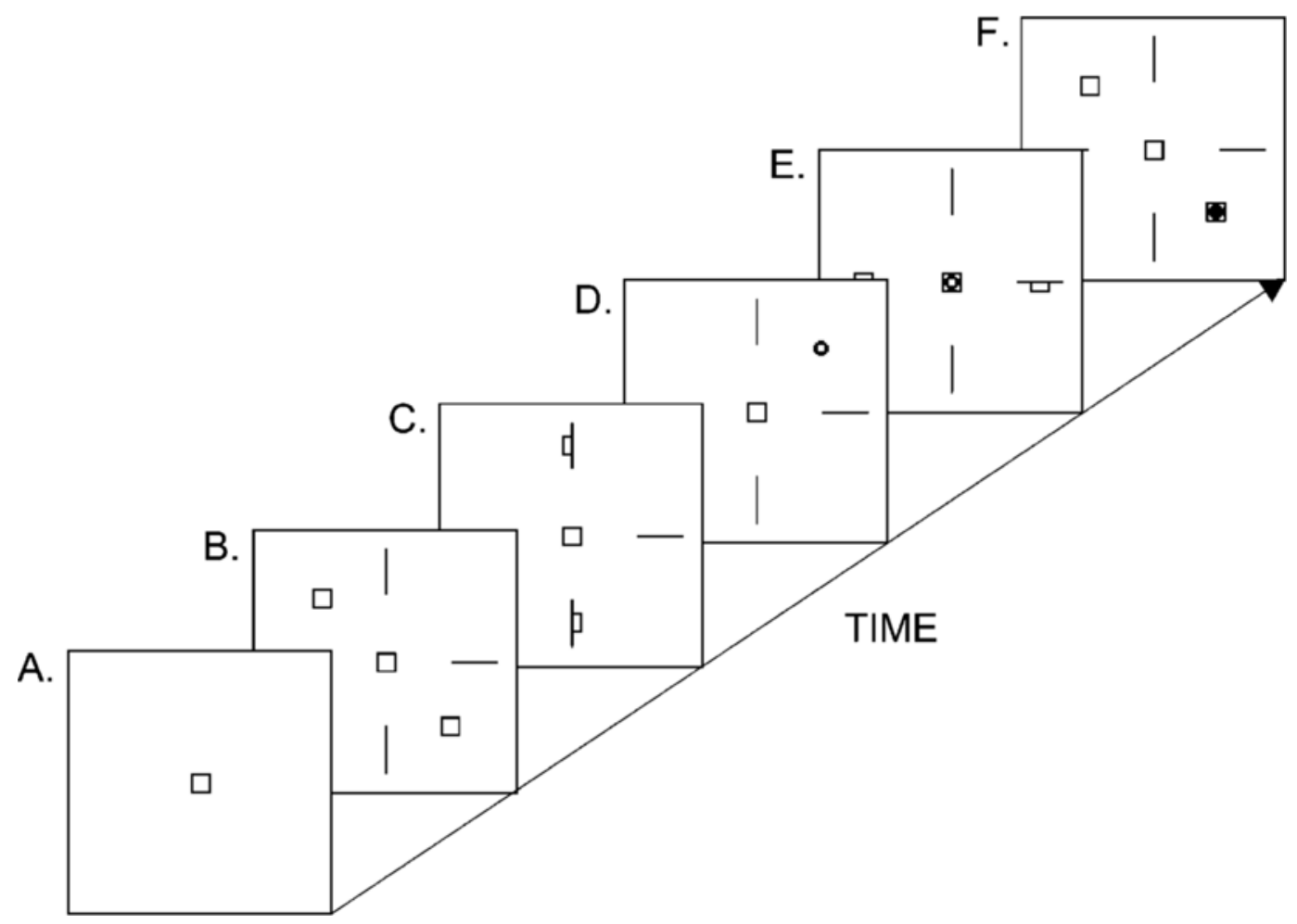

Figure 1. The trial sequence used in Experiment 1. The animation used in the actual experiment contained 33 frames, to ensure smooth motion; only a few critical frames are shown here, to illustrate the key aspects of the task. A sample animation may be viewed over the Internet at http://pantheon.yale.edu/ chun/demos/demoIOR. $\mathrm{html}$. In the real display, the color of the background was black. The outlines of the three squares were dark gray, and the two lines of each occluder were light gray. The peripheral and fixation cues, shown as empty disks in panels $D$ and $E$, were white filled disks. The probe, shown as a black disk in panel F, was colored blue. 
peared behind occluders, accretion and deletion features were shown along the boundaries of the occluders during one frame. Each moving square was totally occluded for $600 \mathrm{msec}$ when it passed behind an occluder.

Procedure. Twenty practice trials were randomly selected from the main experiment. A typical trial is schematized in Figure 1. Each trial began with a fixation square in the center of the screen (panel A). The subject was instructed to fixate his or her gaze on this square throughout each trial without blinking his or her eyes. When the subject initiated the trial by pressing the space bar, two peripheral squares appeared in the centers of two quadrants diagonally opposite each other (panel B). The initial locations of the peripheral squares were randomly selected from the two pairs of diagonal quadrants. Two $90^{\circ}$ occluders were positioned in the remaining quadrants. The peripheral squares began moving clockwise or counterclockw ise around the fixation square after a 1,000 -msec delay and reached the boundaries of the occluders after $320 \mathrm{msec}$ (panel C). Then the peripheral squares disappeared behind occluders, so that they were invisible for $640 \mathrm{msec}$ thereafter. When the peripheral squares arrived behind the center of their occluders, the first (peripheral) cue was presented for $80 \mathrm{msec}$ at one of the two occluded locations (panel D). When the peripheral squares reappeared, the second cue was flashed for $80 \mathrm{msec}$ at the fixation square, to draw attention away from the moving peripheral objects (panel E). Finally, on a third of the trials, ${ }^{1}$ a probe was presented for $80 \mathrm{msec}$ at one of four possible locations: the two locations occupied by the two peripheral squares and the two remaining empty (occluder) locations (panel F). The stimulus onset asynchrony (SOA) between the peripheral cue and the probe was $640 \mathrm{msec}$. The subject pressed the shift key near his or her dominant hand as quickly and accurately as possible whenever he or she detected the probe. A warning tone signaled an error when he or she responded in a probe-absent trial or failed to respond in a probepresent trial.

Design. When the probe was presented (in one third of all the trials), its location was determined by the combination of two variables: cuing and probe type. The cuing factor refers to whether the peripheral cue and the probe locations were consistent (cued condition) or not (uncued condition). The probe type factor refers to whether the probe appeared on an object or on an empty location. Combining the cuing and the probe type factors yielded four conditions: The probe could appear at the cued or the uncued (occluder) location or on the cued or the uncued object. Environmentbased IOR predicts longer RTs to a cued location than to an uncued location, whereas object-based IOR predicts longer RTs to a cued object than to an uncued object. Other variables, such as the direction of orbiting movement and the initial locations of peripheral squares, were fully counterbalanced with the probability of probe presence. The total number of trials was 192, including 64 probepresent trials. The order of the trials was randomized.

\section{Results and Discussion}

The subjects committed false alarms on $0.8 \%$ of the probe-absent trials and made no misses on the probepresent trials at all. RTs on the probe-present trials were sorted according to the four conditions obtained by crossing the two main factors: cuing and probe type. Prior to analysis, RTs beyond three standard deviations from the mean of each condition for each subject were trimmed iteratively until outliers were no longer found. This procedure removed $1.4 \%$ of all the observations. Then mean RTs, calculated from four conditions across subjects, were submitted to a $2 \times 2$ repeated measures analysis of variance (ANOVA).
Figure 2 shows that responses to the probe were slower in the cued condition than in the uncued condition ( $M=374$ and $363 \mathrm{msec}$, respectively) and were slower in the object condition than in the location condition $(M=$ 376 and $360 \mathrm{msec}$, respectively). These differences were confirmed by the ANOVA. The main effect of cuing was significant $\left[F(1,17)=14.22, M S_{\mathrm{e}}=150.35, p<.005\right]$, indicating that IOR occurred in our experiment. The main factor of probe type was also significant $[F(1,17)=$ $\left.27.60, M S_{\mathrm{e}}=165.75, p<.001\right]$, which may be attributed to the possibility that probe detection was masked by the outline of the squares in the object condition. The interaction between cuing and probe type was not significant $\left[F(1,17)=1.49, M S_{\mathrm{e}}=110.74, p>.2\right]$.

Comparisons confirmed both environment-based and object-based IOR. The mean RT was longer in the cued location condition than in the uncued location condition $[M=364$ vs. $356 \mathrm{msec} ; t(17)=2.17, p<.05]$ and, more important, in the cued object condition than in the uncued object condition $[M=383$ vs. $369 \mathrm{msec} ; t(17)=$ $3.49, p<.005] .^{2}$

Our results provide novel evidence that IOR can occur to objects that are invisible behind occluders, which is contrary to what Tipper et al. (1994) found. One critical reason for the discrepancy may be that our display modifications increased the probability that the occluded objects in our experiment served as targets of attentional selection. First, proper occlusion cues may have enhanced the representations of occluded objects (Scholl \& Pylyshyn, 1999). Also, the occluders were less salient and less likely to have been given attention than the occluders in Tipper et al. (1994). Finally, our cuing scheme increased the relevancy of the occluded objects to the task. As such, object-based attention could be directed to the occluded objects even though they did not have any visible features.

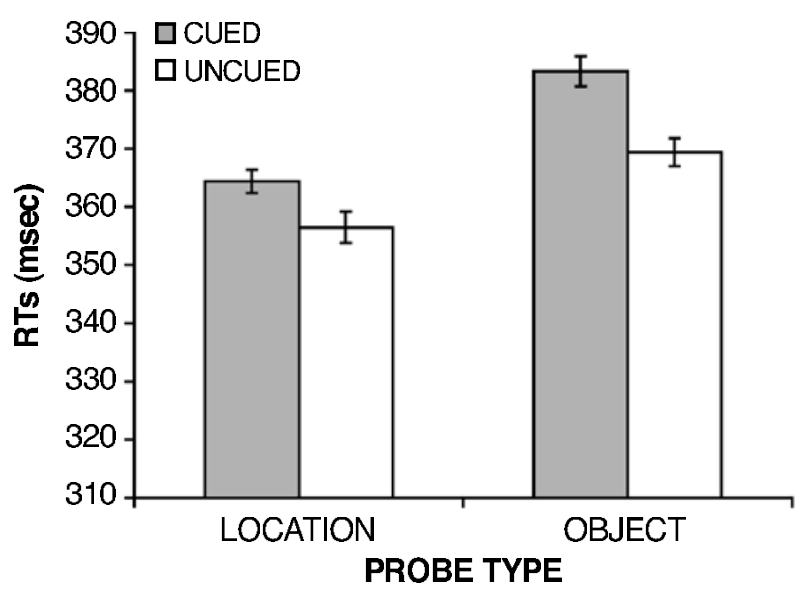

Figure 2. The mean reaction times (RTs) for the correct trials in Experiment 1. Error bars indicate one standard error of the mean after removing between-subjects variance. 


\section{EXPERIMENT 2}

We extended the findings in Experiment 1 by probing the moving objects while they were occluded. If an occluded object maintains an inhibitory tag, responses should be delayed at the location where the cued object is undergoing occlusion. In contrast, if visibility is required to observe IOR to an object, we should not observe delayed responses to the probe of an occluded object.

\section{Method}

Eighteen Yonsei University undergraduates were recruited for course credit. A typical trial is shown in Figure 3. At the beginning of each trial, before the movement began, each square was located on top of the occluder that would later serve to occlude the other square (panel B). The square was thus visible while it moved across the next quadrant (panel C). The first cue appeared on one of the moving squares as they rotated $90^{\circ}$ clockwise or counterclockwise around the center (panel D). The second cue appeared on the fixation square while the moving squares were sliding under the edges of the occluders (panel E). Then the squares disappeared behind the occluders. In a third of the trials at a $640-\mathrm{msec}$ SOA, a probe was presented at one of four possible locations: two locations where squares were undergoing occlusion and two other locations orthogonal to them (panel F). The other conditions and procedures were the same as those in Experiment 1.

\section{Results and Discussion}

The subjects committed false alarms on $0.8 \%$ of the probe-absent trials and misses on $0.3 \%$ of the probe- present trials. RTs on the probe-present trials were sorted according to the four conditions of the two main factors: cuing and probe type. The iterative trimming procedure described in Experiment 1 excluded $2.1 \%$ of all the observations prior to analysis. Then the mean RTs from the four conditions were submitted to a $2 \times 2$ repeated measures ANOVA.

The data for each condition are plotted in Figure 4. Responses were longer in the cued condition than in the uncued condition ( $M=363$ and $341 \mathrm{msec}$, respectively), which was confirmed by the significant main effect of cuing $\left[F(1,17)=11.08, M S_{\mathrm{e}}=203.17, p<.005\right]$. Specifically, the planned comparison showed that RTs were longer in the cued location condition than in the uncued location condition $[M=352$ vs. $346 \mathrm{msec} ; t(17)=3.10$, $p<.01]$ and were longer in the cued object condition than in the uncued object condition $[M=356 \mathrm{vs}$. $343 \mathrm{msec} ; t(17)=2.56, p<.05]$. Neither the main effect of probe type nor the interaction between cuing and probe type was significant $\left[F(1,17)=2.15, M S_{\mathrm{e}}=63.83\right.$, $p>.2$, for probe type; $F(1,17)=0.11, M S_{\mathrm{e}}=80.20, p>$ .8 , for the interaction]. ${ }^{3}$

The results showed that responses were delayed not only to the location where the cue appeared, but also to the "empty" location, where the cued square was currently occluded. The inhibitory bias did not dissipate as the cued square disappeared. Instead, inhibition remained attached to the representation of the occluded square.

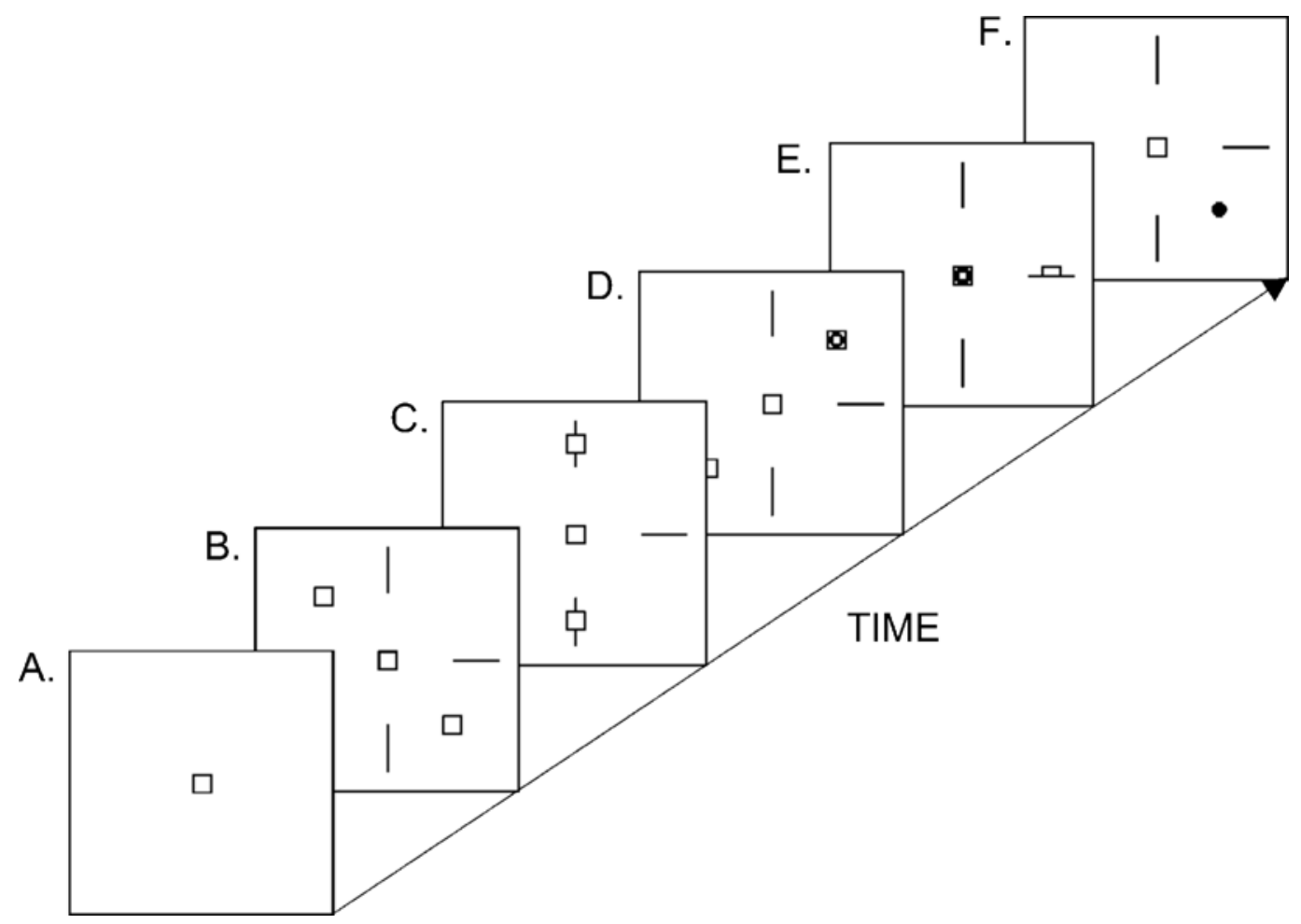

Figure 3. The trial sequence used in Experiment 2. A sample animation may be viewed over the Internet at http://pantheon.yale.edu/ chun/demos/demoIOR.html. 


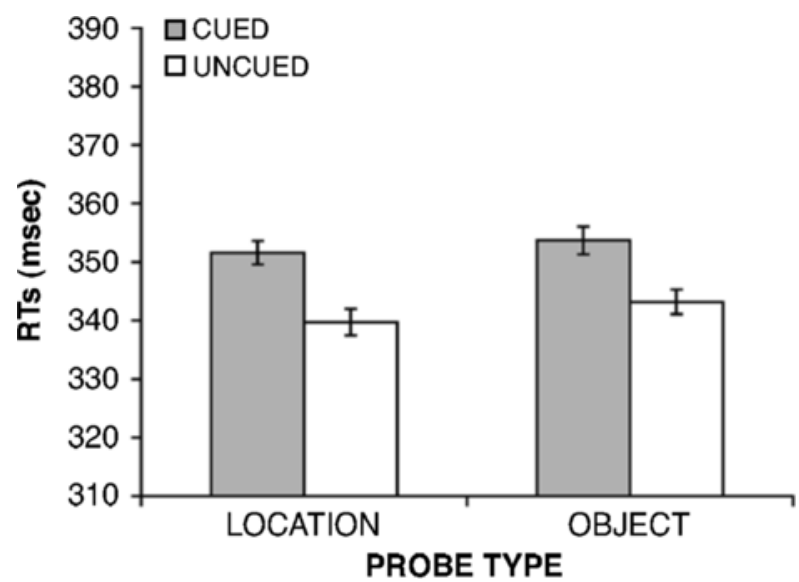

Figure 4. The mean reaction times (RTs) for the correct trials in Experiment 2.

Along with the findings from Experiment 1, these results provide compelling evidence that the visibility of an object is not necessary to observe IOR to it.

\section{EXPERIMENT 3}

We demonstrated object-based IOR to objects that were occluded while they were cued, in Experiment 1, and while they were probed, in Experiment 2. In the final experiment, we tested whether IOR would occur to an object that was occluded when cued and when probed. In this experiment, four occluders with a central angle of $45^{\circ}$ were arranged so as to form a figure $\mathrm{X}$ on the screen. They occluded moving objects during both cue and probe intervals.

\section{Method}

Seventeen Yonsei University undergraduates were recruited for course credit, and 1 graduate student volunteered. Figure 5 shows the trial sequence used in Experiment 3. When the subjects initiated each trial, two moving squares appeared along the vertical or the horizontal axis. Four occluders, with a central angle of $45^{\circ}$, were placed around the fixation box, arranged in a figure $\mathrm{X}$ formation (panel A). Thus, the peripheral cue (panel C) and the probe (panel G) were always presented while the moving squares were occluded. The nearest distance between either the peripheral cue or the probe and the boundary of an occluder was $1.9^{\circ}$. Deletion or accretion cues of the objects sliding under or away from the occluders were animated across three frames (panels B, D, and F). Other conditions and procedures were the same as in Experiment 1.

\section{Results and Discussion}

The subjects committed false alarms on $1.4 \%$ of the probe-absent trials and misses on $0.2 \%$ of the probepresent trials. The iterative trimming procedure excluded $1.9 \%$ of all the observations prior to analysis.

The mean RTs are plotted in Figure 6. Responses to the probe were slower in the cued condition than in the uncued condition $[M=350$ vs. $339 \mathrm{msec} ; F(1,17)=26.21$,

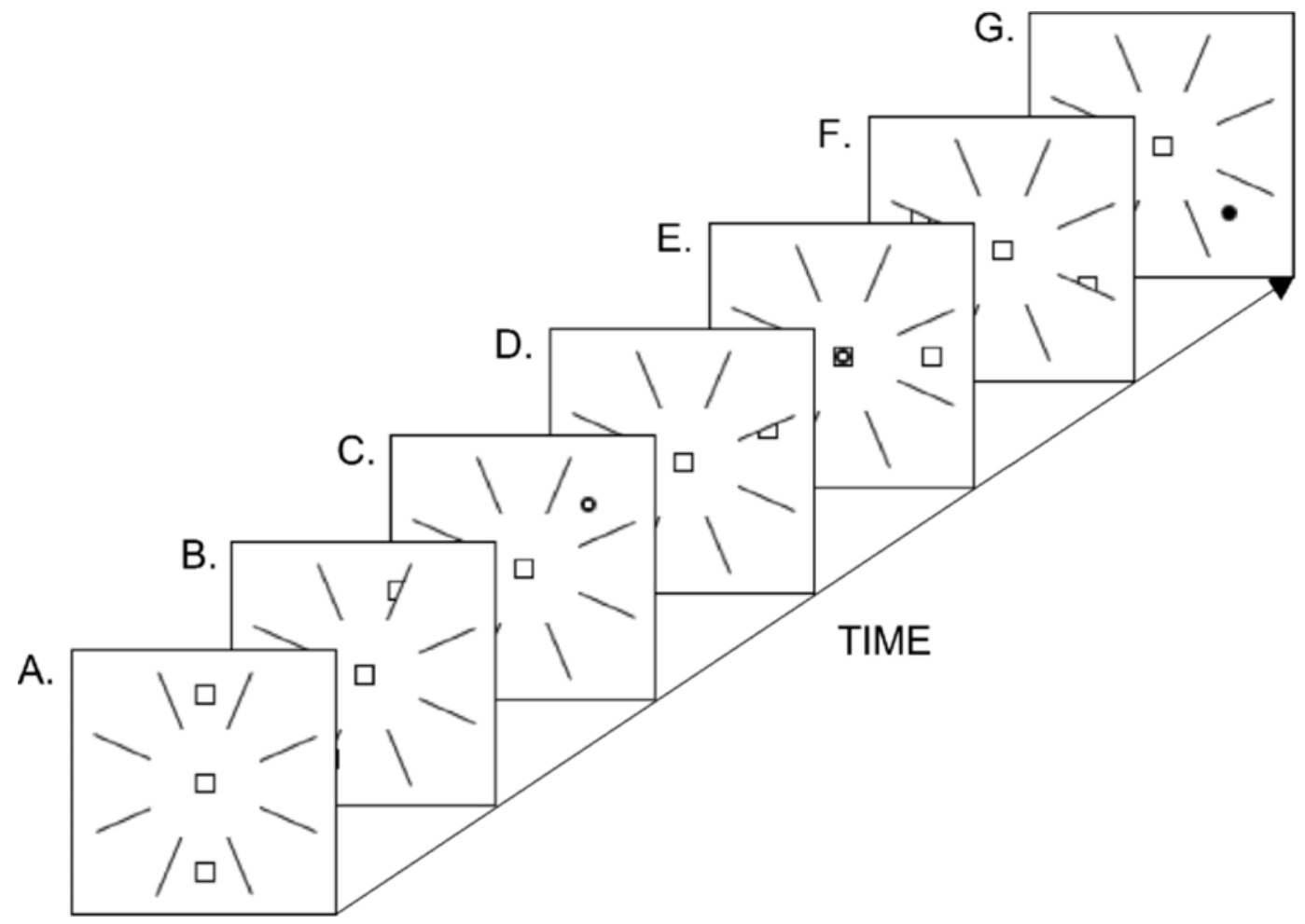

Figure 5. The trial sequence used in Experiment 3. A sample animation may be viewed over the Internet at http://pantheon.yale.edu/ chun/demos/demoIOR.html. 


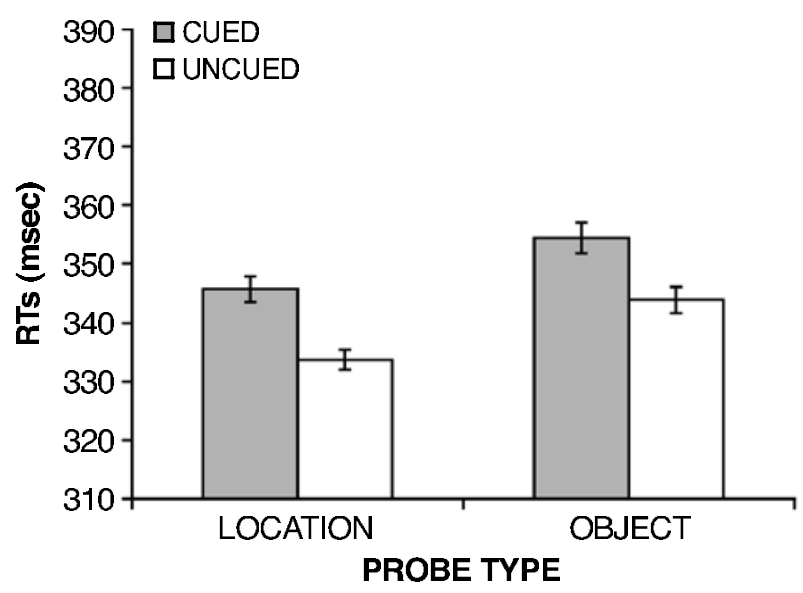

Figure 6. The mean reaction times (RTs) for the correct trials in Experiment 3.

$\left.M S_{\mathrm{e}}=86.51, p<.001\right]$. In planned comparisons, responses were significantly slower in the cued location condition than in the uncued location condition $[M=346$ vs. $334 \mathrm{msec} ; t(17)=5.17, p<.001]$. Responses were also slower in the cued object condition than in the uncued object condition $[M=354$ vs. $344 \mathrm{msec} ; t(17)=$ $2.96, p<.01]$.

Interestingly enough, Figure 6 reveals that responses were slower in the object condition than in the location condition ( $M=349$ and $340 \mathrm{msec}$, respectively). The difference was statistically significant $[F(1,17)=8.36$, $\left.M S_{\mathrm{e}}=192.06, p<.01\right]$. In a post hoc analysis, the difference in RTs between the cued object condition and the cued location condition was marginally significant $[M=$ 354 vs. $346 \mathrm{msec} ; t(17)=2.01, p=.061]$. The difference in RTs between the uncued object condition and the uncued location condition was significant $[M=344$ vs. $334 \mathrm{msec} ; t(17)=3.10, p=.01]$. The interaction between cuing and probe type was not significant $[F(1,17)=0.11$, $\left.M S_{\mathrm{e}}=75.56, p>.8\right] .{ }^{4}$

The results are very similar to those in Experiment 1. First, we observed both environment- and object-based IOR. An inhibitory bias survived the repeated occlusion of the targets. Second, responses were delayed at the locations occupied by the occluded squares, a result that was not expected. Responses should not have been different between the object condition and the location condition, given that the probe appeared in empty (physically identical) locations in both cases. Intriguingly, this response delay for occluded objects appeared to be stable, since we were able to replicate it. ${ }^{5}$

Why were responses slower to occluded objects than to empty locations? One possible account can be derived from the attentional momentum hypothesis of IOR, which predicts that amount of IOR is a function of deviation from the path of attention (Pratt, Spalek, \& Bradshaw, 1999; but see Snyder, Schmidt, \& Kingstone, 2001). In our task, the momentum of attention always started in the direction of the initially cued peripheral location toward the central fixation. From that point, the uncued object condition always required a change in the path of attention toward an orthogonal location, whereas the uncued location did not (it maintained attentional momentum). Accordingly, RTs were delayed to the uncued object, relative to the uncued location. However, this interpretation is undermined by the failure to observe the same result in Experiment 2, which was physically identical to Experiment 3. Thus, attentional momentum does not appear to play a general role in this task.

Another factor to consider is the time needed to access an occluded visual representation. It may take longer for a probe to access an occluded representation than to access a standard location. Alternatively, occluded representations may compete with their occluders, so that the visual system may require time to resolve whether a probe appearing at an occluder location belongs to the occluder or the occluded object. In either case, one may expect slower responses to occluded objects than to empty locations. But this account is not tenable if one considers that such a result did not occur in Experiment 2, which revealed object-based IOR.

One final account arises from the possibility of masking. The probe might be masked by the persisting representation of the occluded object in Experiment 3. Consider motion masking (Yantis \& Nakama, 1998), in which the discrimination of a target was delayed when it was perceived to appear on the path of perceived apparent motion. As such, motion masking predicts slowed responses in the object condition, because the probe appears along the path of motion. This account would have to assume that motion masking is relatively perceptual, so that it did not occur in Experiment 2 because the path of implied motion was twice as long $\left(4.3^{\circ}\right.$ vs. $\left.1.9^{\circ}\right)$. In contrast, object-based IOR survived a $4.3^{\circ}$ spatial gap, since it is cognitive rather than perceptual. Admittedly, this speculative account will require further investigation.

\section{GENERAL DISCUSSION}

It has been argued that IOR encourages the sampling of new information during visual search (Klein, 1988; Klein \& MacInnes, 1999; Posner \& Cohen, 1984). The present study supports this idea by presenting two important observations. First, as was originally reported by Tipper and his colleagues (Tipper et al., 1991; Tipper et al., 1994), RTs for detecting a probe onset were elevated for a novel location, into which a previously cued object had moved. Since the visual environment is not always static, the capability of tracking an inhibited object might be an essential feature of an efficient search mechanism. Second, responses were delayed for the cued object that had been occluded when it was cued (Experiment 1), when it was probed (Experiment 2), or both (Experiment 3 ). This novel finding was arrived at by reducing the salience and relevance of occluders and 
employing compelling occlusion cues (such as accretion and deletion). By showing that the inhibitory tag to an object withstands the spatiotemporal discontinuity due to occlusion, the present study complements the ecological validity of object-based IOR, in a manner consistent with studies on occlusion perception and attention (Moore et al., 1998; Scholl \& Pylyshyn, 1999).

Recently, Haimson and Behrmann (2001) claimed that visual attention does not distinguish between occluded and occluding objects. Their displays presented two pairs of crossed hockey sticks. A transient cue appeared at one end of a stick, followed by a probe. Responses to the probe were faster when the probe appeared at the other end of the cued stick than when it appeared at the equidistant end of the uncued stick, replicating a typical within-object benefit (Egly, Driver, \& Rafal, 1994). Importantly, within-object benefits were maintained even when the other end of the cued stick was occluded by another stick, so that the probe appeared in the overlapped region. Haimson and Behrmann interpreted this result as indicating that attention spread from the occluded object to the overlapping occluder in the same location, due to spatial facilitation. The same mechanism may underlie our experiments in that the occluded moving object was selected by attention that spread down from the cued occluder. However, Haimson and Behrmann's study does not exclude the possibility that attention may be selective for either the occluder or the occluded object in some situations. Alternatively, the probe appearing on the occluder in Haimson and Behrmann's study may have actually accessed the occluded representation in their task. Thus, it is not necessary to presume that attention spread from a cued occluded object to an uncued occluding object in the probed location. Further study is needed to resolve this issue, but our conclusion remains the same: Attention can be allocated to occluded objects.

Although our finding of IOR to an occluded object seemingly contradicts a previous failure to observe it (Tipper et al., 1994, Experiment 6), it is worth noting that the discrepancy may simply reflect different scenarios that are both equally valid. When one is searching the airport for a friend among a group of moving people, a person can be occluded in one of two ways. First, the person could go behind another person, who may also serve as a potential target for action. This new relevant object may capture attention, and accordingly, no IOR would occur to the occluded object, as was demonstrated in Tipper et al.'s (1994) study. In the second scenario, consider a person who is occluded as he or she moves briefly behind a wall, which induces little competition for attention since it is not relevant to the task of finding the friend. In this case, IOR would be expected to the occluded object, as has been demonstrated in our study. As such, both cases can be true: Sometimes occluded objects can be associated with inhibition, but sometimes this is not possible. ${ }^{6}$ In conclusion, our study extends Tipper et al.'s (1994) original finding, and together, the two studies re- veal that IOR may enjoy considerable ecological validity in a broad range of dynamic real-world situations.

\section{REFERENCES}

Abrams, R. A., \& DobKin, R. S. (1994). Inhibition of return: Effects of attentional cuing on eye movement latencies. Journal of Experimental Psychology: Human Perception \& Performance, 20, 467-477.

Brainard, D. H. (1997). The Psychophysics Toolbox. Spatial Vision, 10, 433-436.

DAvis, G., \& Driver, J. (1994). Parallel detection of Kanizsa subjective figures in the human visual system. Nature, 371, 791-793.

Davis, G., \& Driver, J. (1998). Kanizsa subjective figures can act as occluding surfaces at parallel stages of visual search. Journal of Experimental Psychology: Human Perception \& Performance, 24, 169-184.

Egly, R., Driver, J., \& Rafal, R. D. (1994). Shifting visual attention between objects and locations: Evidence from normal and parietal lesion subjects. Journal of Experimental Psychology: General, 113, 501-517.

Gibson, B. S., \& Egeth, H. (1994). Inhibition of return to object-based and environment-based locations. Perception \& Psychophysics, 55, 323-339.

Haimson, C., \& Behrmann, M. (2001). Cued visual attention does not distinguish between occluded and occluding objects. Psychonomic Bulletin \& Review, 8, 496-503.

He, Z I., \& NaKay ama, K. (1992). Surfaces versus features in visual search. Nature, 359, 231-233.

KLEIN, R. M. (1988). Inhibitory tagging system facilitates visual search. Nature, 334, 430-431.

KLEIN, R. M. (2000). Inhibition of return. Trends in Cognitive Sciences, 4, 138-147.

KLeIN, R. M., \& MacInnes, W. J. (1999). Inhibition of return is a foraging facilitator in visual search. Psychological Science, 10, 346352.

MAYLOR, E. (1985). Facilitatory and inhibitory components of orienting in visual space. In M. I. Posner \& O. S. M. Marin (Eds.), Attention and performance XI (pp. 189-203). Hillsdale, NJ: Erlbaum.

Moore, C. M., Yantis, S., \& Vaughan, B. (1998). Object-based visual selection: Evidence from perceptual completion. Psychological Science, 9, 104-110.

Posner, M. I., \& Cohen, Y. (1984). Components of visual orienting. In H. Bouma \& D. G. Bouwhuis (Eds.), Attention and performance X: Control of language processes (pp. 531-556). Hillsdale, NJ: Erlbaum.

Pratt, J., SpaleK, T. M., \& Bradshaw, F. (1999). The time to detect targets at inhibited and noninhibited locations: Preliminary evidence for attentional momentum. Journal of Experimental Psychology: Human Perception \& Performance, 25, 730-746.

Rafal, R. D., Calabresi, P. A., Brennan, C. W., \& Sciolto, T. K. (1989). Saccade preparation inhibits reorienting to recently attended locations. Journal of Experimental Psychology: Human Perception \& Performance, 15, 673-685.

Scholl, B. J., \& Pylyshyn, Z. W. (1999). Tracking multiple items through occlusion: Clues to visual objecthood. Cognitive Psychology, 38, 259-290.

Snyder, J. J., Schmidt, W. C., \& Kingstone. A. (2001). Attentional momentum does not underlie the inhibition of return effect. Journal of Experimental Psychology: Human Perception \& Performance, 27, 1420-1432.

TipPer, S. P., Driver, J., \& Weaver, B. (1991). Short report: Objectcentered inhibition of return of visual attention. Quarterly Journal of Experimental Psychology, 43A, 289-298.

Tipper, S. P., Weaver, B., Jerreat, L. M., \& Burak, A. L. (1994). Object-based and environment-based inhibition of return of visual attention. Journal of Experimental Psychology: Human Perception \& Performance, 20, 478-499.

Yantis, S. (1995). Perceived continuity of occluded visual objects. Psychological Science, 6, 182-186.

YANTIS, S., \& NAKAMA, T. (1998). Visual interactions in the path of apparent motion. Nature Neuroscience, 1, 508-512. 


\section{NOTES}

1. Because the cue-probe SOA was fixed to $640 \mathrm{msec}$ in our study, probes were presented less frequently than in typical detection tasks in which probes were presented with greater temporal uncertainty. We expected that the lower frequency of probe presentation would discourage anticipatory responses. However, probe frequency is not a critical factor, because we replicated the same results even when we doubled the probe frequency (two thirds of the trials; see note 5), which was identical to that used in Tipper et al.'s (1994) experiments.

2 . The averaged medians from untrimmed data were $370 \mathrm{msec}$ in the cued location condition, $361 \mathrm{msec}$ in the uncued location condition, $385 \mathrm{msec}$ in the cued object condition, and $367 \mathrm{msec}$ in the uncued object condition. Statistical outcomes were the same as those with the trimmed data.

3. The averaged medians from untrimmed data were $349 \mathrm{msec}$ in the cued location condition, $338 \mathrm{msec}$ in the uncued location condition, $353 \mathrm{msec}$ in the cued object condition, and $341 \mathrm{msec}$ in the uncued object condition. Statistical outcomes were the same as those with the trimmed data, except for those of the planned comparison between the cued and the uncued object conditions, for which the difference was marginal $[t(17)=2.044, p=.057]$.

4. The averaged medians from untrimmed data were $346 \mathrm{msec}$ in the cued location condition, $336 \mathrm{msec}$ in the uncued location condition,
$353 \mathrm{msec}$ in the cued object condition, and $340 \mathrm{msec}$ in the uncued object condition. Statistical outcomes were the same as those with the trimmed data.

5. In the replication of Experiment 3, 18 undergraduate students at Vanderbilt University volunteered for course credit. The stimuli were presented on iMac computers, which were controlled by MATLAB equipped with psychophysics toolbox extensions. The cue-probe SOA was $688 \mathrm{msec}$. The design was the same as that in Experiment 3, except that a probe showed up on two thirds of 288 trials. Besides both environment- and object-based IOR $[p s<.05]$, we observed that responses were slower in the object condition than in the location condition $[M=312$ vs. $\left.300 \mathrm{msec} ; F(1,17)=20.58, M S_{\mathrm{e}}=135.31, p<.001\right]$. In planned comparisons, responses were slower in the cued object conditions than in the cued location condition $[M=318$ vs. $304 \mathrm{msec} ; t(17)=4.93, p<.001]$ and were slower in the uncued object condition than in the uncued location condition $[M=307$ vs. $295 \mathrm{msec} ; t(17)=2.89, p<.01]$.

6 . We thank Steven Tipper for providing us with this useful insight and example.

(Manuscript received November 19, 2002; revision accepted for publication May 6, 2003.) 